\title{
Integrating Approximation and Interactive Decision Making in Multicriteria Optimization
}

\author{
KATHRIN KLAMROTH \\ Institute of Applied Mathematics, University of Erlangen-Nuremberg \\ Martensstr. 3, D-91058 Erlangen, Germany \\ email: klamroth@am.uni-erlangen.de \\ KAISA MIETTINEN \\ Helsinki School of Economics \\ P.O. Box 1210, FI-00101 Helsinki, Finland \\ email: miettine@hse.fi
}

\begin{abstract}
We present a new interactive hybrid approach for solving multicriteria optimization problems where features of approximation methods and interactive approaches are incorporated. We produce rough approximations of the nondominated set and let the decision maker indicate with the help of reference points where to refine the approximation. In this way, (s)he iteratively directs the search towards the best nondominated solution. After the decision maker has identified the most interesting region of the nondominated set, the final solution can be fine-tuned with existing interactive methods. We suggest different ways of updating the reference point as well as discuss visualizations that can be used in comparing different nondominated solutions. The new method is computationally relatively inexpensive and easy to use for the decision maker.

Subject classifications: Programming: Multiple Criteria. Nonlinear. Interactive Methods. Reference Point

Area of review: Optimization
\end{abstract}

\section{Introduction}

In multicriteria optimization, the problem is to find the best compromise solution in the presence of several conflicting criteria, see, for example, Ballestero and Romero (1998), Chankong and Haimes (1983), Cohon (1978), Hwang and Masud (1979), Miettinen (1999), Sawaragi et al. (1985), Statnikov and Matusov (1995), 
Steuer (1986) and Szidarovszky et al. (1986). In such problems, there is no single well-defined optimal solution but several mathematically equally good solutions, so-called nondominated, noninferior or Pareto optimal solutions can be identified. To be able to find the best among them, that is, the final solution, we need additional preference information from a human decision maker who knows the problem domain.

Methods developed for solving multicriteria optimization problems can be classified according to the role of the decision maker in four classes, see, for example, Hwang and Masud (1979) and Miettinen (1999). If there is no decision maker available, the final solution is some neutral compromise. In a priori methods, the decision maker first specifies preferences and hopes and after that a solution satisfying these hopes as well as possible is identified. The drawback here is that the decision maker may have too optimistic hopes and the final outcome may be a disappointment or it may be otherwise difficult for the decision maker to specify hopes in advance. On the other hand, in a posteriori methods a representation of the set of nondominated solutions is first generated and then the decision maker is supposed to select the most satisfactory solution. The difficulty here is how to display the large amount of data to the decision maker and how to support her/him in finding the best of them. Furthermore, generating a large set of nondominated solutions may be computationally expensive for complicated real-life problems. A possibility to overcome the above-mentioned weaknesses is to use interactive methods, where the decision maker actively takes part in the iterative solution process and specifies preference information gradually. In this way, the decision maker can learn about the problem, its possibilities and limitations as well as the interdependencies among the criteria and possibly even change one's mind about what is desirable. Because only such nondominated solutions are generated that are interesting to the decision maker, interactive methods are generally computationally much less expensive than, for example, a posteriori methods.

When the decision maker iteratively directs the search for the most satisfactory solution in interactive methods, it may be advisable and helpful to first get a rough overview of what the nondominated set looks like, in other words, what kind of solutions are feasible. After that, it is easier to specify preferences and locate the most preferred solution. An example of such an approach where a visualization tool based on interactive decision maps (see Lotov et al. (2004)) and an interactive classification-based NIMBUS method (see Miettinen and Mäkelä (1999), Miettinen (1999), Miettinen and Mäkelä (2000) and Miettinen and Mäkelä (2006)) are hybridized is given in Miettinen et al. (2003). However, that kind of a hybrid approach is best suited for problems with less than five criteria.

In this paper, we present a new general interactive hybrid approach for solving multicriteria optimization problems where we combine ideas of a posteriori approximation methods with an interactive method. The motivation is to benefit from the strengths of these methods and, at the same time, avoid their weaknesses 
discussed above. First we produce a rough approximation of the nondominated set by generating a relatively small representative subset of nondominated solutions. The accuracy of this approximation can be specified by the decision maker by bounding, for example, the number of solutions generated or the approximation error. By studying the approximation, the decision maker can learn about the problem and then we let her/him indicate where to refine the approximation. In this way, (s)he iteratively directs the search towards the best nondominated solution and does not have to compare too many solutions at a time. Piecewise linear approximations that we use provide a powerful and computationally efficient tool to give an overview of what the set of nondominated solutions looks like. By specifying least acceptable values for different criteria in the form of a reference point the decision maker can zoom in or out in the nondominated set and locate the most satisfactory region. Finally, the solution selected as the most preferred one can be given as a starting point to some interactive method for fine-tuning, if desired.

The rest of this paper is organized as follows. In Section 2 we give a problem formulation and introduce the main concepts and notation used. Piecewise linear approximations of the nondominated set are briefly introduced in Section 3 and Section 4 is devoted to the new interactive hybrid approach as well as discussion related to it. The issue of updating the reference point is considered in Section 5, and a numerical example is discussed in Section 6. Finally, the paper is concluded in Section 7.

\section{Problem Definition and Notation}

We consider the following general multicriteria optimization problem (MOP)

$$
\begin{array}{ll}
\max & \left\{z_{1}=z_{1}(x)\right\} \\
& \vdots \\
\max & \left\{z_{n}=z_{n}(x)\right\} \\
\text { s.t. } & x \in X,
\end{array}
$$

where $X \subseteq \mathbb{R}^{m}$ is the feasible set and $z=\left(z_{1}, \ldots, z_{n}\right)^{T}: X \rightarrow \mathbb{R}^{n}$ is the vector of objective functions with individual real-valued objectives $z_{i}(x): X \rightarrow \mathbb{R}$, $i=1, \ldots, n$. A solution $\bar{x} \in X$ is called efficient or Pareto optimal if there is no other solution $x \in X$ with $z(x) \geq z(\bar{x})$, that is, $z_{i}(x) \geq z_{i}(\bar{x})$ for all $i=1, \ldots, n$ and $z(x) \neq z(\bar{x})$. Thus, efficient solutions are those solutions that cannot be improved in all criteria without deterioration in at least one of the criteria. We denote the set of all efficient solutions by $E$. The image $z(\bar{x})$ of an efficient solution $\bar{x} \in X$ in the objective space $\mathbb{R}^{n}$ is called a nondominated solution or a nondominated criterion vector. The set of all nondominated solutions is denoted by $N=z(E)$. 
Unless explicitly stated, we do not make any assumptions on the objective functions and the constraint set of (1) other than assuming the existence of efficient solutions and boundedness of the set of nondominated solutions. That is, we consider convex as well as non-convex and discrete problems, and we do not restrict the number of criteria.

We define the set of all feasible criterion vectors as $Z=\left\{z \in \mathbb{R}^{n}: z=\right.$ $z(x), x \in X\}=z(X)$. In order to ensure the existence of efficient solutions (i.e., $E \neq \emptyset)$ we assume that the set $Z$ is $\mathbb{R}_{\leqq}^{n}$-closed, that is, the set $Z+\mathbb{R}_{\leqq}^{n}$ is closed and that $Z \neq \emptyset$.

The following notation is used throughout the paper. We denote components of vectors by subscripts and enumerate vectors by superscripts. Let $u, w \in \mathbb{R}^{n}$ be two vectors. The notation $u>w$ means that $u_{i}>w_{i}$ for all $i=1, \ldots, n$ whereas $u \geq w$ denotes that $u_{i} \geq w_{i}$ for all $i=1, \ldots, n$, but $u \neq w$. On the other hand, the notation $u \geqq w$ allows equality. The symbols $<, \leq, \leqq$ are used accordingly. We denote the non-positive orthant by $\mathbb{R}_{\leqq}^{n}:=\left\{x \in \mathbb{R}^{n}: x \leqq 0\right\}$. The set $\mathbb{R}_{\geqq}^{n}$

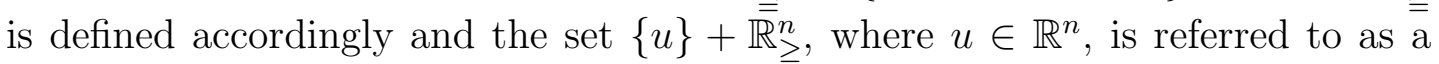
dominating cone. Similarly, for a set $Z \subset \mathbb{R}^{n}$, we use the notation $Z \geqq Z+\mathbb{R}_{\geqq}^{n}$.

The set of properly nondominated solutions is defined according to Geoffrion (1968): A point $\bar{z} \in N$ is called properly nondominated, if there exists $M>0$ such that for each $i=1, \ldots, n$ and each $z \in Z$ satisfying $z_{i}>\bar{z}_{i}$ there exists an index $j \neq i$ with $z_{j}<\bar{z}_{j}$ and

$$
\frac{\bar{z}_{i}-z_{i}}{z_{j}-\bar{z}_{j}} \leq M
$$

Otherwise $\bar{z} \in N$ is called improperly nondominated. Moreover, a point $\bar{z} \in Z$ is called weakly nondominated if there does not exist $z \in Z$ with $z<\bar{z}$. The corresponding solutions $\bar{x} \in X$ are called properly efficient, improperly efficient and weakly efficient, respectively.

The point $\bar{z}$ with components $\bar{z}_{i}=\min \left\{z_{i}(x): x \in E\right\}, i=1, \ldots, n$, is called the nadir point. Note that, while this point is easily found for bicriteria problems, its computation is in general hard if $n>2$ (see, for example, Miettinen, 1999).

By a reference point we mean a point in the criterion space $\mathbb{R}^{n}$. Note that it does not have to be in $Z$. Typically, it consists of desirable or acceptable criterion values for each criterion and is specified by a decision maker, a person who knows the problem considered well and is able to specify preference information related to it.

Throughout the paper, we use the following convex bicriteria example problem taken from Schandl et al. (2001), which was rescaled here to better serve the 
illustrational purpose:

$$
\begin{array}{ll}
\max & z_{1}(x)=x_{1}+x_{2}-5 \\
\max & z_{2}(x)=\frac{1}{5}\left(10 x_{1}-x_{1}^{2}+4 x_{2}-x_{2}^{2}\right)-2.2 \\
\text { s.t. } & 3 x_{1}+x_{2}-12 \leq 0 \\
& 2 x_{1}+x_{2}-9 \leq 0 \\
& x_{1}+2 x_{2}-12 \leq 0 \\
& x \in \mathbb{R}_{\geqq}^{2} .
\end{array}
$$

\section{Approximation of the Nondominated Set}

In the interactive hybrid method that we shall describe in more detail in Section 4, partial approximations of the nondominated set are used to support the decision maker's search for the most preferred solution. Iteratively improved piecewise linear approximations of the nondominated set can be a powerful tool to obtain an overview of the feasible alternatives available at relatively low computational cost. Many different approximation approaches could be embedded in such an interactive procedure, for example, the Estimate Refinement method (see Lotov et al., 2004) or some of the approximation approaches reviewed in Ruzika and Wiecek (2005).

For our method, we suggest the application of an adaptive approximation procedure as described in Klamroth et al. (2002) since it is particularly well-suited for guided refinements based on variations of the reference point suggested by the decision maker, and can thus be nicely embedded in interactive algorithms. It generates piecewise linear approximations in a problem-dependent way, utilizing polyhedral distance functions to construct the approximation and evaluate its quality. The functions automatically adapt to the problem structure and scaling, and thus quickly adapt to the currently investigated region of the nondominated set. This makes the approximation phase unbiased and self-driven.

While the approximation approach used is closely related to several other approximation methods (particularly in the case of convex MOPs), like the NISE method (Cohon, 1978) and the Estimate Refinement method (Lotov et al., 2004), its distinguishing feature lies in the fact that the quality of the current approximation is measured based on the approximation itself and not, for example, by the Hausdorff distance. Consequently, the point that is considered "worst approximated" by a given approximation and that should be added to the approximation in the next iteration may differ from the other methods. This leads in general to different approximation results, even if the individual scalarized subproblems used are of the same or of a similar type. The main advantage of using a problem-dependent distance measure can be seen in the scale-independence achieved which is particularly important in an interactive procedure where the 
reference point may be moved around more or less freely, and where the decision maker may put more emphasis on some of the objectives.

Let us next describe the global approximation procedure of Klamroth et al. (2002) in brief first for convex and then for non-convex problems. For further details, we refer also to Klamroth and Tind (2006).

We start with the case where $Z$ is $\mathbb{R}_{\leqq}^{n}$-convex, that is, $Z+\mathbb{R}_{\leqq}^{n}$ is convex, with int $Z \neq \emptyset$, and suppose that a reference point $\hat{z}^{0}$ is given that satisfies $\hat{z} \in Z_{\leq}$. For a polyhedral gauge $\gamma: \mathbb{R}^{n} \rightarrow \mathbb{R}$, consider the problem

$$
\begin{array}{ll}
\max & \gamma\left(z(x)-\hat{z}^{0}\right) \\
\text { s.t. } & z(x) \in \hat{z}^{0}+\mathbb{R}_{\geqq}^{n} \\
& x \in X .
\end{array}
$$

Let $\gamma$ be defined by a symmetrical unit ball $B$ (i.e., $\gamma(z):=\inf \{\lambda \in \mathbb{R}: z \in \lambda B\}$ ) centered at the origin (which can be assumed to coincide with the reference point), that is obtained by symmetrically extending a given piecewise linear inner approximation of $N$ in the criterion space. Then problem (3) finds a feasible point $z(\bar{x}) \in Z$ that maximizes the problem-dependent $\gamma$-distance from the current approximation in the criterion space (see Figure 1 for an example).

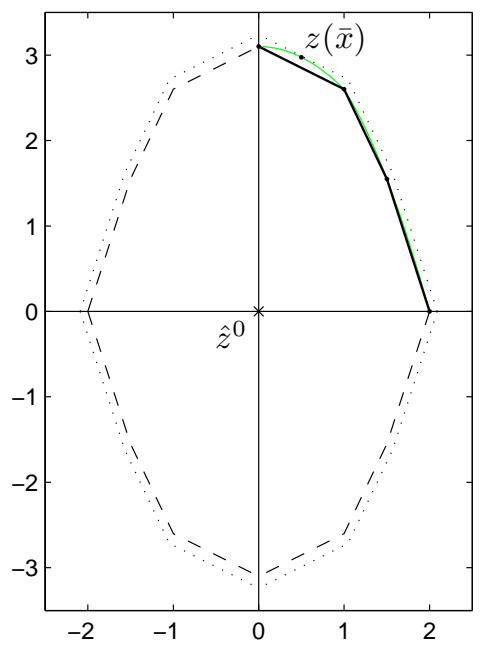

Figure 1: Illustration of an oblique norm applied to problem (2). For this oblique norm, $\bar{x}$ with image $z(\bar{x})$ is an optimal solution of problem $(3)$.

Schandl et al. (2002a) showed that such norms $\gamma$ are oblique norms, that is, they are absolute (in other words, $\gamma(w)=\gamma(u)$ for all $w \in R(u):=\{w \in$ $\mathbb{R}^{n}:\left|w_{i}\right|=\left|u_{i}\right|$ for all $\left.\left.i=1, \ldots, n\right\}\right)$ and they satisfy $\left(z-\mathbb{R}_{\geqq}^{n}\right) \cap \mathbb{R}_{\geqq}^{n} \cap \partial B=$ $\{z\}$ for all $z \in(\partial B)_{\geqq}$. 
Moreover, the solution of problem (3) always is nondominated, and if the reference point additionally satisfies $N \subseteq \hat{z}^{0}+\mathbb{R}_{\geqq}^{n}$, then in fact any properly nondominated solution can be found by solving problems of type (3) with different oblique norms (see again Schandl et al., 2002a). Note that while the first statement remains true for any reference point $\hat{z}^{0} \in Z_{\leq}$, the second statement must be weakened in this case. It can be easily derived from Schandl et al. (2002a) that only those parts of $N$ located in $\hat{z}^{0}+\mathbb{R}_{\geq}^{n}$ can be generated from a fixed reference point by varying the unit ball $B$ of the oblique norm $\gamma$ :

\section{Corollary 1.}

(i) If $\gamma$ is an oblique norm with a reference point $\hat{z}^{0} \in Z_{\leqq}$, then every optimal solution $\bar{x}$ of (3) is an efficient solution of (1).

(ii) If $Z$ is $\mathbb{R}_{\leqq}^{n}$-convex and if $\bar{x}$ is a properly efficient solution of (1) in $\hat{z}^{0}+\mathbb{R}_{\geqq}^{n}$, then there exists an oblique norm $\gamma$ with reference point $\hat{z}^{0}$ such that $\bar{x}$ solves (3).

Problem (3) can be decomposed into multiple subproblems, each of a particularly simple structure. For this purpose, let $B$ be the unit ball of $\gamma$ and denote by $C_{1}, \ldots, C_{s}$ the fundamental cones of $B \cap \mathbb{R}_{\geq}^{n}$. If $d^{j}$ is the normal vector of the facet of the cone $C_{j}, j=1, \ldots, s$, then (3) can be decomposed into $s$ subproblems $\left(P^{j}\right), j=1, \ldots, s$, of the form

$$
\begin{aligned}
& \lambda_{j}=\max d^{j}\left(z(x)-\hat{z}^{0}\right)=\sum_{i=1}^{n} d_{i}^{j}\left(z_{i}(x)-\hat{z}_{i}^{0}\right) \\
& \text { s.t. } \quad x \in X
\end{aligned}
$$

from which the maximum value of $\lambda_{j}, j=1, \ldots, s$, must be selected to obtain an overall optimal solution of (3). Note that each subproblem (4) corresponds to a weighted-sums scalarization of the multicriteria optimization problem (1) and contains only the problem dependent constraints $x \in X$.

The approximation algorithm suggested in Klamroth et al. (2002) now iteratively solves problem (3) by computing optimal solutions of all newly generated subproblems of the form (4), starting from an initial approximation that can be generated, for example, by solving $\max \left\{z_{i}(x): x \in X\right\}$ for all $i=1, \ldots, n$.

In each iteration, the point of worst approximation with respect to the current distance measure $\gamma$ is added to the current approximation by using an appropriate convex hull algorithm (for example the Beneath-Beyond Algorithm, see Edelsbrunner, 1987), see Schandl et al. (2002b) for further details. This leads to an adaptive update of the polyhedral gauge $\gamma$ and thus to the generation of a new set of active subproblems (4) in the updated cones. Figure 2 illustrates the procedure for problem (2). Observe that, for example, in iteration 3 (3rd picture in Figure 2), the added point is not furthest away from the current approximation with respect to the Hausdorff distance. 

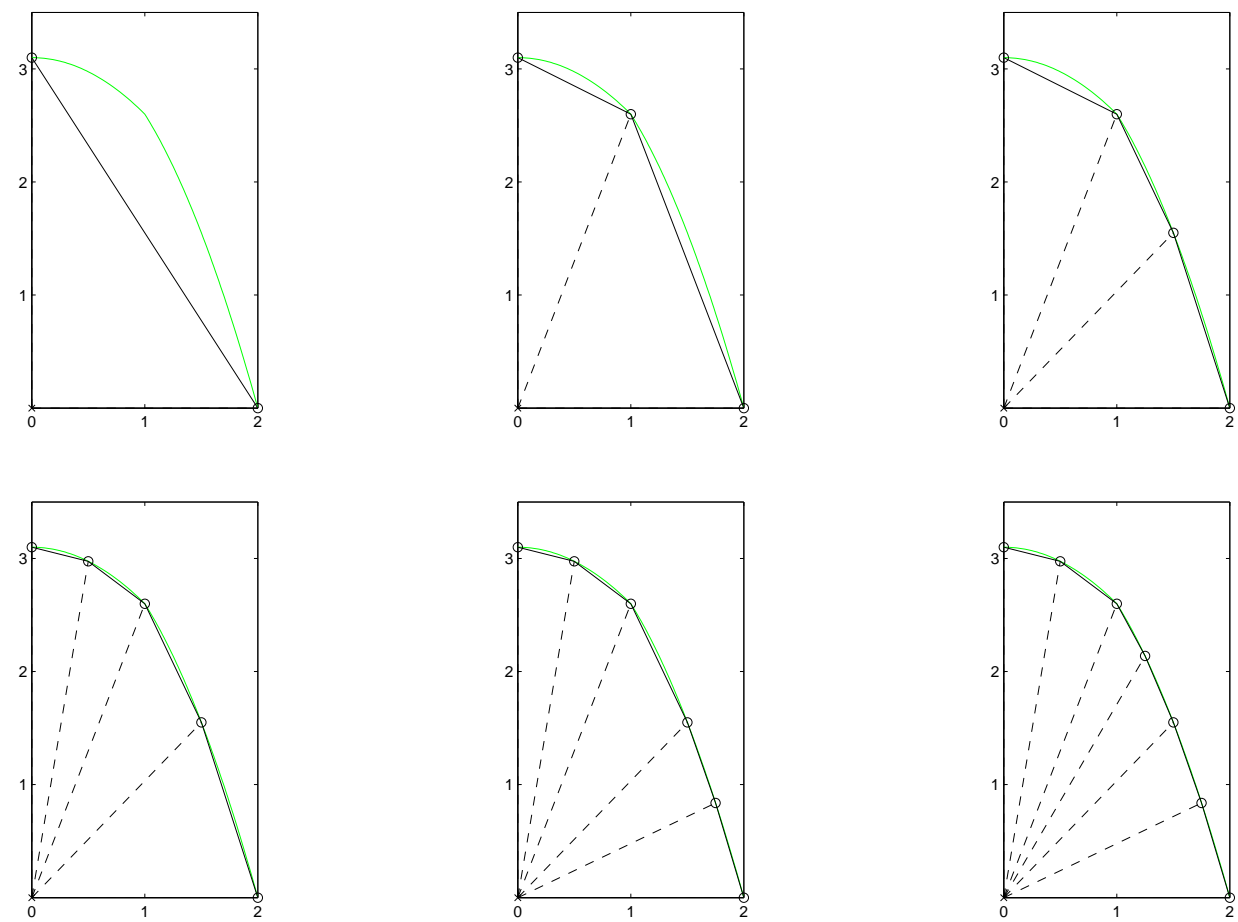

Figure 2: Inner approximation algorithm applied to problem (2)

Note that in the bicriteria case each iteration of the approximation algorithm involves the solution of only two weighted-sums scalarizations (4) of problem (1). Even though the number of active subproblems per iteration may theoretically be larger for $n \geq 3$, it can be expected not to have a considerable impact on the average time needed to find the next iterate. The computational complexity of solving each of these subproblems depends on the objective functions and constraints in question as well as on the single-criteria optimizer used. However, the solution of weighted-sums scalarizations is in general of the same computational complexity as the solution of a single-criteria problem of the same type.

The accuracy of the approximation after $k$ iterations can be bounded based on general convergence results for the approximation of convex bodies with respect to Hausdorff distances by Rote (1992), Kamenev (1992), Kamenev (1994) and Lotov et al. (2004). We obtain a convergence rate of $O\left(k^{-\frac{2}{n-1}}\right)$ for the approximation error with respect to adaptive gauge distances, where $n \geq 2$ is the number of criteria in (1) (see Klamroth and Tind, 2006, for further details).

For non-convex and discrete problems, that is, problems where the set $Z$ is not $\mathbb{R}_{\leqq}^{n}$-convex, the method described above has to be modified with respect to the approximation constructed and the formulation of appropriate scalarized subproblems as described in Klamroth et al. (2002) and Klamroth and Tind 
(2006). However, the main idea of using an adaptive distance measure, based on a reference point and on the current approximation, to asses the approximation quality is transferred also to this case.

For the choice of the reference point $\hat{z}^{0}$ we again have to assume that $\hat{z}^{0} \in$ $Z_{\leqq}=Z+\mathbb{R}_{\leqq}^{k+1}$. Since the nondominated set $N$ may now be disconnected, a piecewise linear approximation can only aim at approximating the set $N_{c}:=$ $\left\{z \in Z_{\leqq}: \nexists \tilde{z} \in Z_{\leqq}\right.$s.t. $\left.\tilde{z} \geq z\right\}$. Similarly, the convex unit ball of a distance measuring gauge $\gamma$ is replaced by a non-convex set $B$ that is constructed from the intersection of dominating cones. The set $B$ is then used as a unit set to define a distance measuring function $\gamma$ as $\gamma(z):=\inf \{\lambda \in \mathbb{R}: z \in \lambda B\}$.

The basic idea for an approximation procedure is - similar to the convex case - to minimize the maximum $\gamma$-distance between a nondominated point in $Z$ and the boundary of $B$. However, since weighted-sums scalarizations are not suitable for general non-convex problems (see, for example, Miettinen, 1999), in this case variants of the Chebyshev method are used for the generation of candidate points which are iteratively added to the approximation. This ensures that all solutions generated are nondominated and that any nondominated solution can be found by varying $B$ appropriately. The candidate that is worst approximated with respect to the $\gamma$-distance is selected for addition and included in the approximating set $B$ together with all points in the intersection of its domination cone with the positive orthant.

The computational complexity of this approach again strongly depends on the complexity of solving one of the scalarized Chebyshev subproblems which in general can be a more time-consuming task than solving convex problems of the type (4). Since we do not want to restrict our discussion to some specific class of problems, we cannot give a general bound on this complexity.

In each iteration, at most $n$ of the single-criteria scalarized Chebyshev problems have to be solved. As was shown in Klamroth and Tind (2006) the approximation error, measured with respect to the adaptive distance measure $\gamma$, converges to zero with an increasing number of iterations.

\section{Interactive Approach with Reference Points}

In this section we introduce our interactive hybrid approach utilizing piecewise linear approximations of the nondominated set. Approximations provide information to the decision maker about the problem and serve as the basis for refinements of the decision maker's preferences, which can then be used to identify preference regions in which the approximation has to be refined.

In Section 3, we assumed that the reference point $\hat{z}^{0} \in \mathbb{R}^{n}$ was fixed. But if we allow the reference point to change, for example, by moving the reference point closer to a region of interest for the decision maker, the approximation can be refined in specific areas identified by the decision maker. In this way we 
construct our interactive hybrid procedure. It is important that, when compared to ordinary approximation methods, we can decrease computational costs because we do not consider the whole nondominated set with equal emphasis, but the approximation is made more accurate only in those regions the decision maker really is interested in.

\subsection{The Impact of the Reference Point}

By allowing the decision maker to specify the reference point, it can be used to

- capture preference information of the decision maker in the form of minimal criterion values that should be attained,

- and zoom the approximation in the region of interest to the decision maker.

Since the reference point has an immediate impact on the relative size of the unit ball of the approximating gauge $\gamma$, moving $\hat{z}^{0}$ closer to the nondominated set will lead to a relative increase of the distance of the approximation from the nondominated set in this area.

In order to obtain meaningful problem formulations (3) (or corresponding ones in the non-convex case), the choice of the reference point should be restricted to the set $Z_{\leqq}$. We can distinguish three different situations, where we denote the reference point of the current iteration of the decision process by $\hat{z}^{i}$, and that of the next iteration by $\hat{z}^{i+1}$ :

- $\hat{z}^{i} \leqq \hat{z}^{i+1}$ : In this case the decision maker has identified a region of interest from the previous approximation and wishes to refine the approximation in the area defined by $\left(\hat{z}^{i+1}+\mathbb{R}_{\geqq}^{n}\right) \cap Z_{\leqq}$(zooming in).

- $\hat{z}^{i} \geqq \hat{z}^{i+1}$ : The decision maker wishes to explore a larger region surrounding the previously approximated part of the nondominated set (zooming out).

- Otherwise, the decision maker prefers to explore a (partially) different region of the nondominated set without using the information gathered in the previous iterations.

Because the decision maker can freely study different nondominated solutions and get to know the problem, it may be useful to save interesting solutions for later consideration during the search process. This may be particularly useful if the decision maker wants to learn more about the problem and explore different regions of the nondominated set as mentioned above in the third item. 


\subsection{Interactive Algorithm}

Let us denote the set of saved solutions by $A$. The main steps of the interactive hybrid algorithm can be given as follows:

1. Set $i=0$ and $A=\emptyset$. Ask the decision maker to specify the number of solutions $P$ to be shown to her/him at each iteration.

2. Ask the decision maker to select a reference point $\hat{z}^{i} \in Z_{\leqq}$.

3. Construct a (rough) approximation of $\left(\hat{z}^{i}+\mathbb{R}_{\geqq}^{n}\right) \cap Z_{\leqq}$consisting of $P$ solutions, that is, criterion vectors.

4. Show the approximation to the decision maker. If the decision maker wishes to save some of the solutions, add them in $A$.

5. If some of the points in the approximation or in $A$ is acceptable and desirable for the decision maker as a final solution, stop. Otherwise, if the decision maker would like to continue with a reference point or classification based interactive method in the current neighborhood, take the components of the current reference point as bounds for the criteria and continue with another method. Otherwise, go to step (2) and let the decision maker update $P$ if so desired.

Let us next discuss some details of the algorithm. In the first iteration, it is possible to use the worst possible criterion values as the components of the reference point so that the decision maker gets a rough approximation of the whole nondominated set to start with. For this purpose, the criterion values calculated as $\min \left\{z_{i}(x): x \in X\right\}$, for all $i=1, \ldots, n$, (if they exist) can be used as the first reference point. Alternatively, the nadir point can be used if it is available. Otherwise, the decision maker can be asked to specify the smallest acceptable values for each criterion and then these values are used as the components of the first reference point.

It is up to the decision maker to choose the number $P$ of nondominated solutions (s)he wants to see in each iteration before updating the reference point. The more solutions there are, the more accurate the approximation gets but, on the other hand, the more difficult it is for the decision maker to study them, and generating the approximation will be computationally more expensive. Since in an $n$-criteria problem the individual function maximizers (extremal solutions) define in general $n$ solutions, the value of $P$ could be reasonably selected as some positive multiple of $n$. Alternatively, if the decision maker wants to specify a desired accuracy of the approximation, the number of solutions to be generated can be obtained based on the relative approximation error.

Let us emphasize that the value of $P$ does not need to remain fixed throughout the whole solution process. The decision maker may always change the number 
of solutions to be calculated in the approximation during the solution process if so desired. An important feature making a difference between ordinary approximation approaches and our interactive method is that the approximation is only refined in regions that are specified by the decision maker. For this reason, comparably few points may be sufficient for the decision maker to get an idea of the feasible alternatives. Interruptions of the approximation process and later refinements are also possible.

Different visualization tools can be used to help the decision maker in comparing the different solutions in the approximation. Examples of visualizations for three nondominated solutions involving five criteria are given in Figure 3. This figure illustrates bars charts, bars in three dimensions, value paths, spider web charts and petal diagrams (see, for example, Miettinen (1999), Miettinen (2003) and Trinkaus and Hanne (2005)). By having different options available the decision maker may choose which types of visualizations (s)he finds most informative.

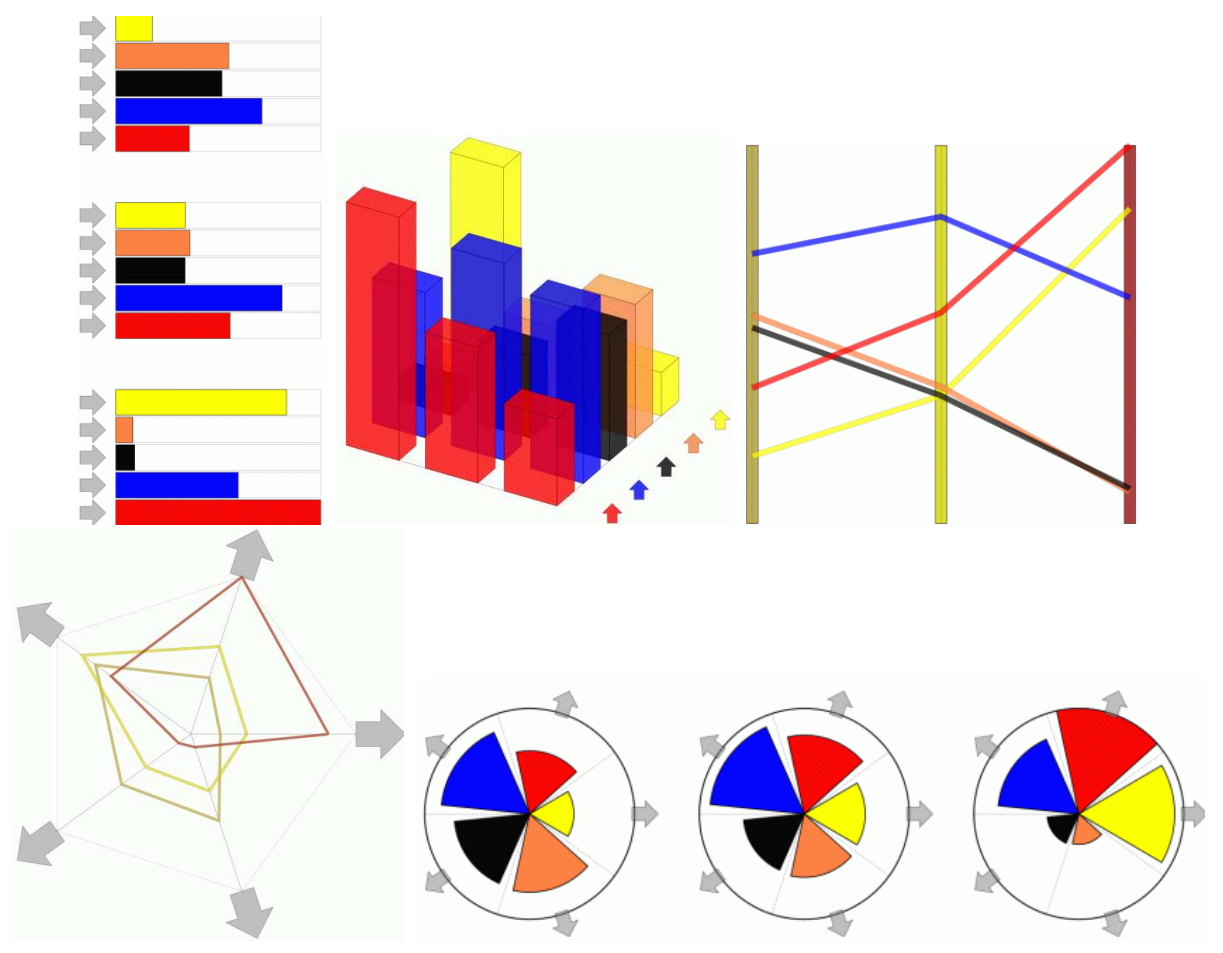

Figure 3: Different visualization possibilities

It is also possible to arrange the nondominated criterion vectors in a decreasing order according to some of the criteria in order to help the decision maker to find the most interesting ones. When studying the different solutions, the decision maker can drop uninteresting solutions and filter out some of the solutions by specifying upper or lower bounds or interesting intervals for criterion values. With 
the same visualization tools the decision maker can also study the set $A$ where (s)he has saved interesting solutions.

After the decision maker has identified a desirable region in the set of nondominated solutions, (s)he can select one of the points in the last approximation as the final solution. Alternatively, (s)he can start an interactive reference point or classification based method so that the bounds specified in the components of the reference point of the last approximation are included as constraints in the problem. In this case, the decision maker can work with the real problem and not the approximation to fine-tune the solution by specifying her/his hopes in the form of a reference point consisting of desirable values for each criterion or in the form of a classification indicating what kind of changes would make the current solution even better. Thanks to the extra constraints set on the criterion values, the consideration now concentrates on the region found interesting in the preceding phase done with approximations. Note that in this phase, the reference point can be any point in the criterion space. Examples of reference point based methods include the reference point method by Wierzbicki (1986) and reference direction approach by Korhonen and Laakso (1986) whereas classification based method include the satisficing trade-off method by Nakayama (1995) and the NIMBUS method by Miettinen (1999) and Miettinen and Mäkelä (2006).

\section{Updating the Reference Point}

After having studied the current approximation, the decision maker may have in mind values of the different criteria (s)he wants to use as the components of the next reference point. If the decision maker wants to zoom in, in other words, wants to specify a reference point closer to the nondominated set, it is possible to show the current reference point to the decision maker and then (s)he can indicate for which criteria and how much higher new values would be acceptable to form the next reference point. Correspondingly, zooming out can be carried out by specifying lower values than in the current reference point. Naturally, the decision maker can specify any criterion values as the components of the next reference point irrespectively of what the current reference point is. But if the decision maker wants to zoom in or zoom out in the nondominated set, there are different possibilities how to support the decision maker in specifying the next reference point, that is, new minimal acceptable values for the criteria.

\subsection{Guiding the Search by Selecting Nondominated Solu- tions}

One possible way to help the decision maker in specifying a new reference point is to present to her/him a reasonably small set of candidate points as a new reference point. For this purpose, suppose that the current approximation with 
the reference point $\hat{z}^{i} \in Z_{\leqq}$is defined by its normal vectors $d^{1}, \ldots, d^{s} \in \mathbb{R}^{n}$, and the approximation consists of the solutions $z\left(x^{1}\right), \ldots, z\left(x^{P}\right) \in N$. Appropriate candidates for the next reference point $\hat{z}^{i+1} \in Z_{\leqq}$would then, for example, be the points

$$
z^{(i+1), j}:=\hat{z}^{i}+\beta \cdot\left(z\left(x^{j}\right)-\hat{z}^{i}\right), \quad j=1, \ldots, P
$$

with a refinement parameter $\beta<1$ that may also be selected by the decision maker. Note that by setting $\beta$ to be positive, we move closer to the nondominated set, that is, zoom in. On the other hand, we can zoom out by using a negative value.

Let us assume that the decision maker has identified the most satisfactory solution $z\left(x^{j}\right)$ among the ones in the current approximation, that is, $z\left(x^{1}\right), \ldots, z\left(x^{P}\right)$ $\in N$. Then, in order to further explore the (variable-size) neighborhood of this solution, the corresponding point $z^{(i+1), j}$ could be selected to serve as the reference point $\hat{z}^{i+1}$ for the next iteration of the procedure.

Let us next assume that the decision maker wants to zoom in. Once a corresponding new reference point has been chosen, the approximation is filtered to leave in the approximation only the solutions located in the new approximation area given by $\left(\hat{z}^{i+1}+\mathbb{R}_{\geqq}^{n}\right) \cap Z_{\leqq}$such that the respective extremal solutions (maximizing the individual criteria in the reduced feasible set) become a part of the approximation. This can be realized, for example, by performing a direction search according to Pascoletti and Serafini (1984) along the coordinate directions $e^{j}, j=1, \ldots, n$, starting at the new reference point $\hat{z}^{i+1}$ :

$$
\begin{array}{ll}
\operatorname{lexmax} & \left(\alpha, \sum_{j=1}^{n} q_{j}\right) \\
\text { s.t. } & z(x)=\hat{z}^{i+1}+\alpha e^{j}+q \\
& q \in \mathbb{R}_{\geqq}^{n} \\
& x \in X,
\end{array}
$$

where the lexicographic maximization includes the term $\sum_{j=1}^{n} q_{i}$ as a second function to be optimized to avoid weakly nondominated solutions.

If necessary, that is, if the number of solutions in the resulting approximation is below the pre-specified number $P$, the approximation is refined by calculating a new approximation with reference point $\hat{z}^{i+1}$. If function evaluations are less costly, the approximation can be recomputed completely in the specified area. Otherwise, the solutions of the existing approximation may be included in the next iteration to save function evaluations. In other words, the old approximation within the reduced feasible set is augmented in the latter case. Given the next approximation, the refinement process is continued as described in the interactive algorithm in Subsection 4.2.

On the other hand, if the decision maker wants to zoom out by specifying a negative $\beta$ in (5) and wants to keep the number of solutions in the approximation 
equal to the pre-specified value of $P$, then (s)he has to delete some of the solutions in the existing approximation, for example, with the help of the visualization tools. After that, as many new solutions are added to the approximation with the new reference point. On the other hand, the decision maker can increase $P$ and then as many new points are added to the approximation as needed. If so desired, the solutions maximizing the criteria individually in the new feasible set can be added in the approximation as in the case of zooming in.

The search may be diversified by storing all candidates for possible reference points from the previous iterations and allowing the decision maker to select one of them (at certain stages of the procedure) as the next reference point.

Figure 4 shows an example of the above-described ideas of generating a new reference point in problem (2). Here, the decision maker decided to zoom in at each iteration.
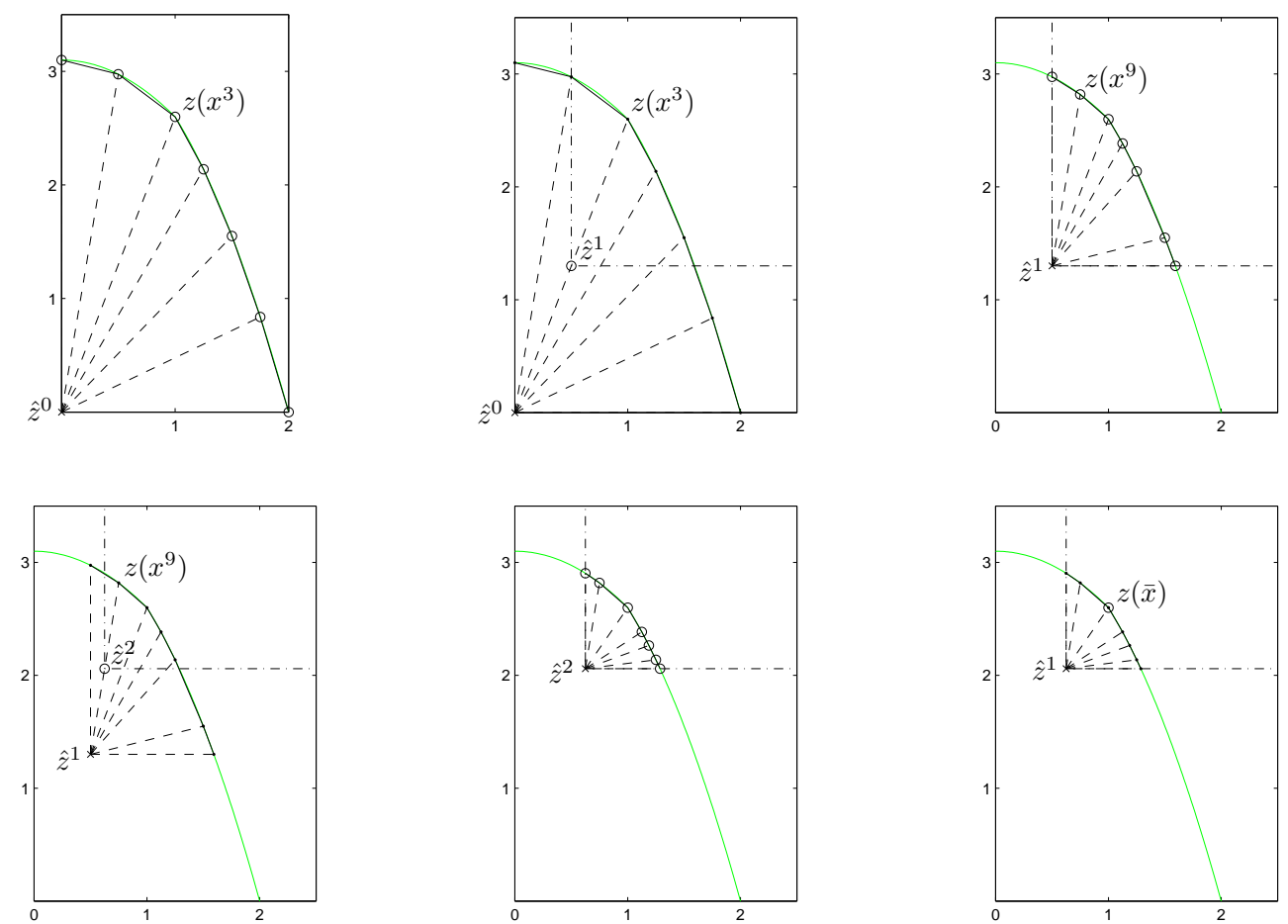

Figure 4: Updating the reference point using candidate reference points

In Figure 4, we set $P=7$, in other words, we assume that the approximation is sufficiently fine as soon as it has seven solutions. Given the initial approximation of Figure 2 (last picture) obtained with the nadir point $\hat{z}^{0}$ used as a reference point, suppose that the point $z\left(x^{3}\right)$ is chosen by the decision maker as the most preferred solution among the generated nondominated points $z\left(x^{1}\right), \ldots, z\left(x^{7}\right) \in$ $N$. Setting $\beta=\frac{1}{2}$, the reference point for the next stage of the procedure is 
$\hat{z}^{1}=\hat{z}^{0}+\frac{1}{2}\left(z\left(x^{3}\right)-\hat{z}^{0}\right)$, see the 2 nd picture in Figure 4 . The approximation is updated and refined in the resulting approximation area (see the 3rd picture in Figure 4). After having it extended by computing three new points so that again seven solutions are given in the refinement area (reusing old points whenever possible), a new reference point is requested from the decision maker. Selecting the point $z\left(x^{9}\right)$ for the next refinement step and setting $\beta=\frac{1}{2}$ leads to the next reference point $\hat{z}^{2}=\hat{z}^{1}+\frac{1}{2}\left(z\left(x^{9}\right)-\hat{z}^{1}\right)$ (see the fourth picture in Figure 4), from which the approximation is again updated and refined (see the 5th picture in Figure 4). At this point the decision maker may be satisfied with the given alternatives and select, for example, the point $z(\bar{x})$ as the most preferred solution, see the last picture in Figure 4.

The final approximation obtained in Figure 4 is shown in Figure 5 without the nondominated set (which actually is unknown in real problems).

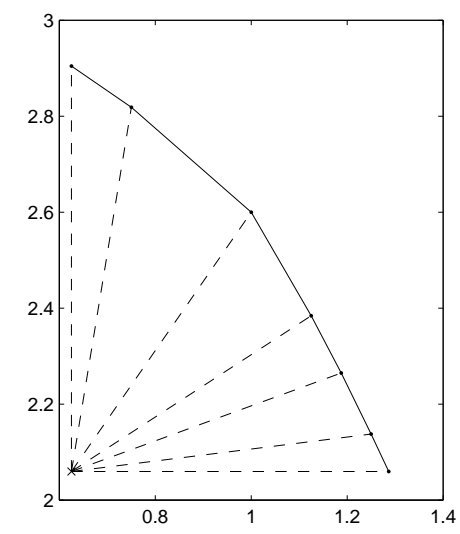

Figure 5: Approximation obtained after two refinement steps

\subsection{Guiding the Search by Selecting Lower Bounds}

Using bounds to refine the search for the most preferred solutions was already suggested by Fandel (1972) and will here be embedded into a sequential refinement of approximations as described in Subsection 4.2.

If the decision maker wants to zoom in, as an alternative to the approach described in the previous subsection, the decision maker could be asked to give a rank order for the criteria, for example, according to how interesting they are (which may differ from one iteration to the other). Then the components of the next reference point are specified in this order.

Let us assume that the ranking corresponds to the numbering of the criteria. Then the solutions $z\left(x^{1}\right), \ldots, z\left(x^{P}\right)$ forming the current approximation are presented to the decision maker, and (s)he selects from these points some solution 
$z\left(x^{j}\right), j \in\{1, \ldots, P\}$ to fix the lower bound as $b_{1}:=z_{1}\left(x^{j}\right)$ for the first criterion. The next bound (for the second criterion) is selected using a subset of the solutions in the current approximation. This subset is of the form

$$
\left\{z\left(x^{k}\right): z_{1}\left(x^{k}\right) \geq b_{1}, k=1, \ldots, P\right\},
$$

and after having a look at it the decision maker selects the lower bound for the second criterion. This procedure is repeated until lower bounds $b_{i}$ have been obtained for all $i=1, \ldots, n$. Smaller and smaller subsets corresponding to (7) are shown to the decision maker in order to ensure that the bounds obtained are feasible in the sense that the resulting vector $b=\left(b_{1}, \ldots, b_{n}\right)^{T}$ lies in $Z_{\leqq}$. If the subset to be shown decreases too fast, the decision maker can take a step backward and select a looser bound for the previous criterion.

Consequently, the new reference point from which the approximation is recomputed and refined (cf. Subsection 5.1) is now given by $\hat{z}^{i+1}:=\left(b_{1}, \ldots, b_{n}\right)^{T} \in Z_{\leqq}$. Figure 6 illustrates the selection of a new reference point and consecutive refinements of the approximation when the current approximation and its subsets are used to get bounds for the new reference point for problem (2). The decision maker may then, for example, select the solution $z(\bar{x})$ as the most preferred solution.
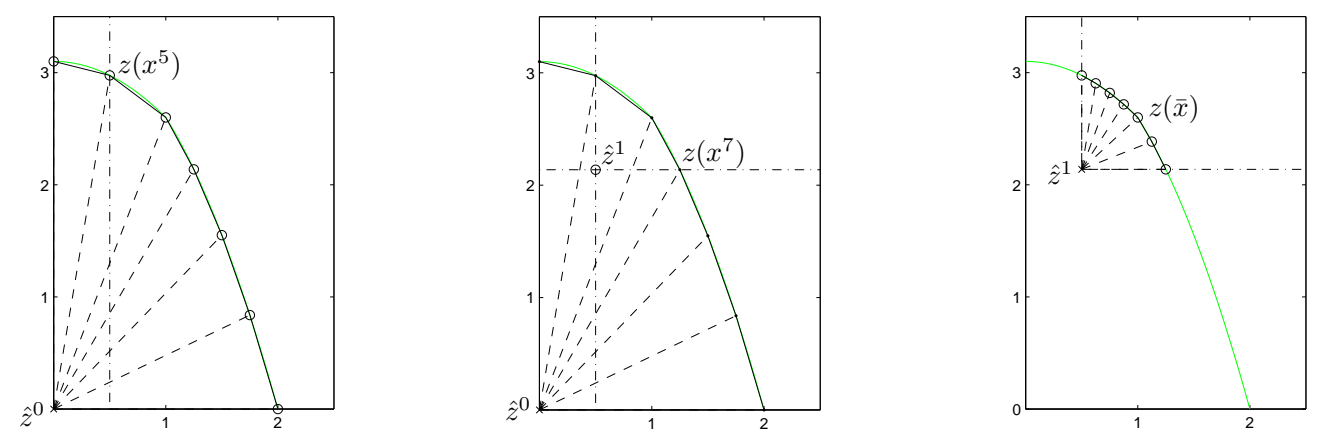

Figure 6: Updating the reference point based on bounds

The approximation obtained (without the nondominated set in the background) is depicted in Figure 7.

\subsection{Guiding the Search Using Classification}

One more possibility to support the decision maker in specifying the next reference point is to use classification. This means that the current reference point is shown to the decision maker and (s)he is asked to classify the criteria into those where the current minimal acceptable level should be

- increased from the current one, 


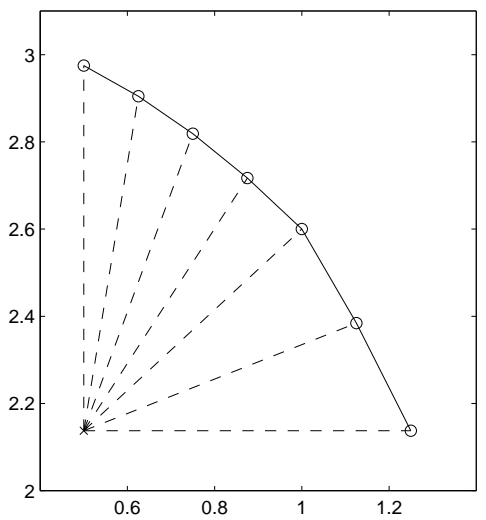

Figure 7: Approximation obtained after one iteration (by selecting bounds)

- decreased from the current one or

- kept the same.

Besides classification, the decision maker is also supposed to set the new levels in the first two classes. In order to avoid infeasibility, this choice should be constrained such that the new reference point still satisfies $\hat{z}^{i+1} \in Z_{\leqq}$. For example, the desired improvement in the respective criteria could be approximated by moving a steplength $\lambda$ towards the corresponding criteria values of a feasible solution in the current approximation (cf. Subection 5.1). At the same time, the reference point may be moved away by a step length of $\bar{\lambda}$ from another feasible solution.

The next reference point is then formed using the new as well as the current values (if the last class is also used) for the appropriate criteria. This resembles the classification used in interactive methods for directing the search for the most satisfactory solution, see, for example, Miettinen (1999) and Miettinen and Mäkelä (2006).

When the new reference point has been found, the current approximation is once again filtered so that only the solutions in the new approximation area $\left(\hat{z}^{i+1}+\mathbb{R}_{\geqq}^{n}\right) \cap Z_{\leqq}$are retained. Using only the first and the last class means zooming in and using the last two classes means zooming out and in those cases recomputing and refining the approximation can be done as described in Subsection 5.1. Otherwise, if the new approximation area does not contain any of the solutions of the current approximation the whole approximation is to be recomputed (with the new reference point). Figures 8 and 9 show an example of the application of this approach. 

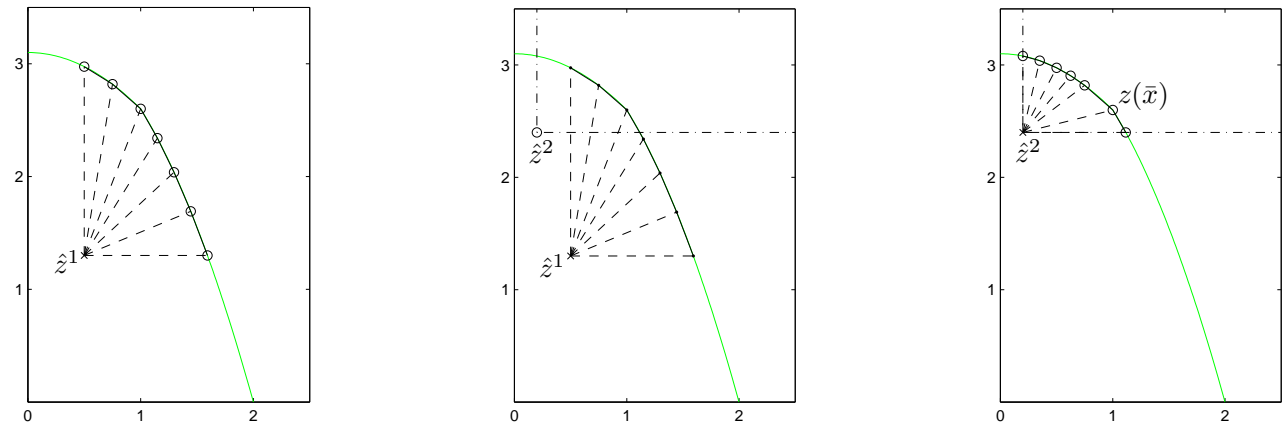

Figure 8: Updating the reference point based on classification

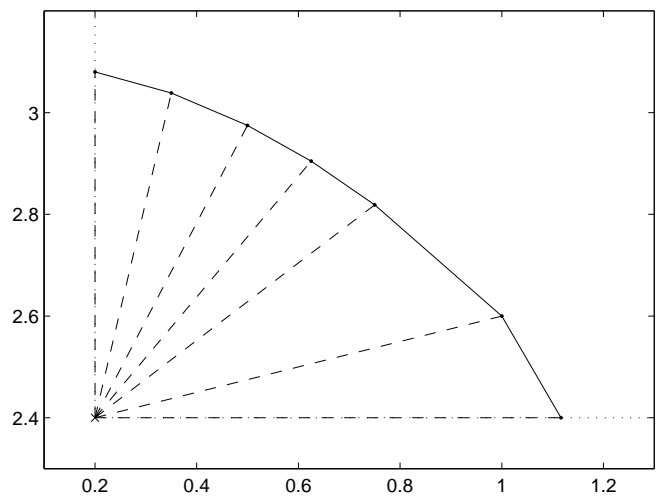

Figure 9: Approximation obtained after one iteration (using classification)

\section{Numerical Example}

To illustrate how our interactive hybrid method can be applied for problems with more criteria, the bicriteria example problem (2) was extended by three objective functions as follows:

$$
\begin{array}{ll}
\max & z_{1}(x)=x_{1}+x_{2}-5 \\
\max & z_{2}(x)=\frac{1}{5}\left(10 x_{1}-x_{1}^{2}+4 x_{2}-x_{2}^{2}\right)-2.2 \\
\max & z_{3}(x)=\left(x_{1}-5\right)\left(x_{2}-11\right) \\
\max & z_{4}(x)=-\left(3 x_{1}+x_{2}-12\right) \\
\max & z_{5}(x)=x_{2} \\
\text { s.t. } & 3 x_{1}+x_{2}-12 \leq 0 \\
& 2 x_{1}+x_{2}-9 \leq 0 \\
& x_{1}+2 x_{2}-12 \leq 0 \\
& x \in \mathbb{R}_{\geqq}^{2}
\end{array}
$$


This problem is nonconvex. Note that objectives 4 and 5 model a situation where the decision maker wants to maximize functions on which bounds are set in the constraints as well. This situation frequently occurs in practice when the role of objective functions and constraints is interchangeable. All numerical results were obtained using an implementation of the approximation method described in Section 5 in Matlab 7.0.4 by Raith (2005).

We first consider a problem consisting of the first three objectives given in problem (8). This allows us to use three dimensional graphical illustrations of the approximations generated. Starting with an approximation of the nadir point as the first reference point (that was computed using a pay-off table), and bounding the number $P$ of points generated during one approximation step by 50 , we obtain the image shown in Figure 10.

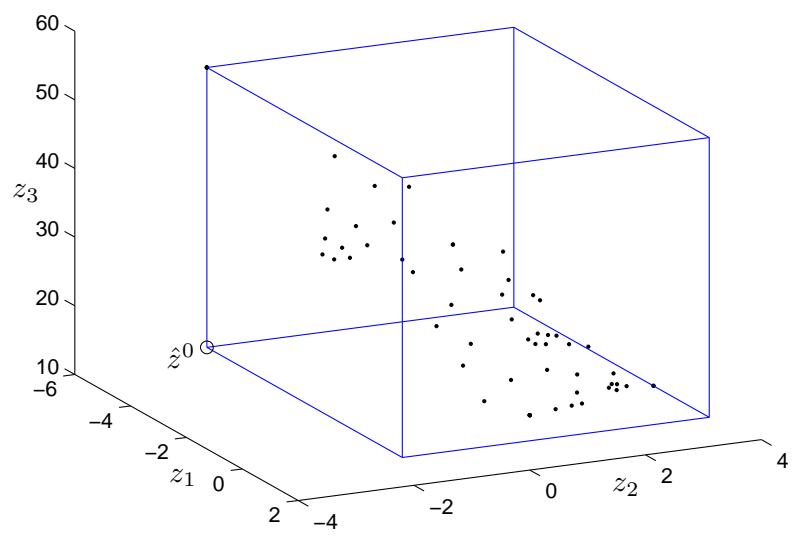

Figure 10: Initial approximation of problem (8) with 50 nondominated points, considering only the first three objectives

Selecting the first $12(=3 \cdot 4)$ solutions of this approximation (sorted with respect to the first objective) for an illustration using value paths (cf. Figure 3 ), we obtain the result illustrated in Figure 11. Note that all the value paths to be shown here have been scaled with the smallest and highest values of the criteria in the set of points in question. Magnitudes in question are best revealed by showing numerical values to the decision maker as well. It is also possible that the decision maker sets a desirable scaling for the objectives. Let us point out that the decision maker can select any number of solutions to be visualized. (Because of the way the approximation is generated, using more points means including points in areas where the approximation is least accurate.)

For the next iteration the decision maker may select, for example, the fifth solution of Figure 11 (from left) and zoom in as described in Section 5.1, using a zooming factor of $\beta=\frac{1}{2}$. The recomputed approximation is illustrated for 50 


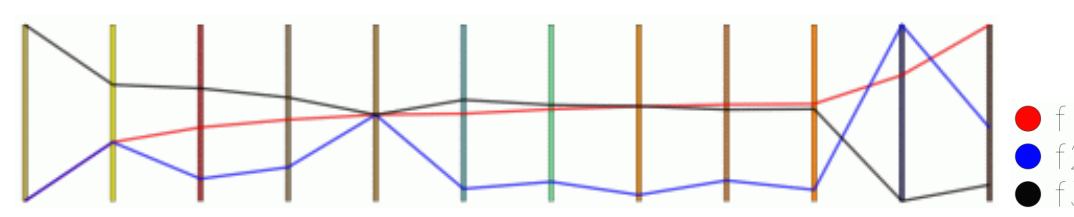

Figure 11: The first 12 nondominated points that were generated in Figure 10, illustrated by value paths

nondominated points in Figure 12. Figure 13 shows value paths for the first 12

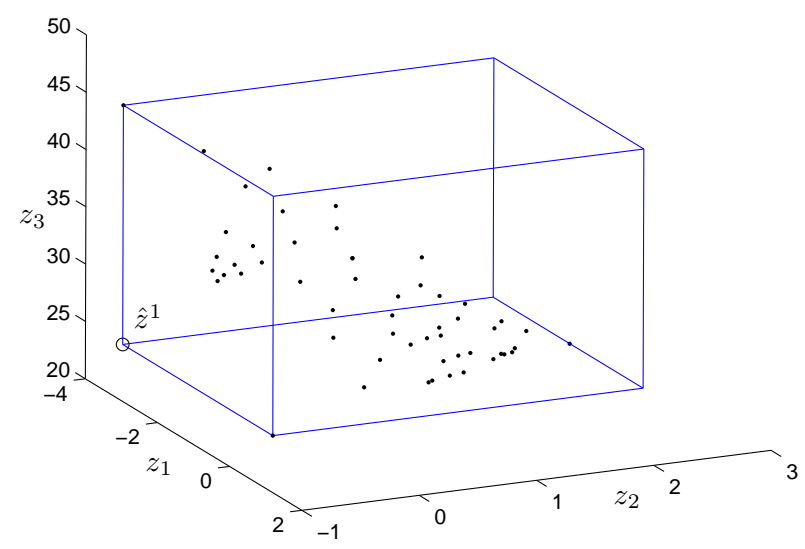

Figure 12: Second approximation of problem (8) with 50 nondominated points, considering only the first three objectives

solutions generated.

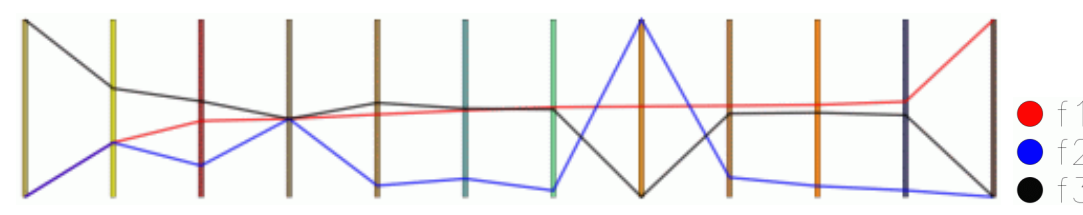

Figure 13: The first 12 nondominated points that were generated in Figure 12, illustrated by value paths

Now the decision maker may prefer to select lower bounds on the individual objectives (cf. Section 5.2) so that the bound on the first criterion (-1.4531) is selected based on solution 4 , on the second (-0.7003) based on solution 5 , and on the third (34.0375) based on solution 9 in Figure 13 (from left). Thus, the new reference point is $\hat{z}^{2}=(-1.4531,-0.7003,34.0375)^{T}$. The approximation was again recomputed (see Figure 14) and the first 12 solutions are illustrated using 


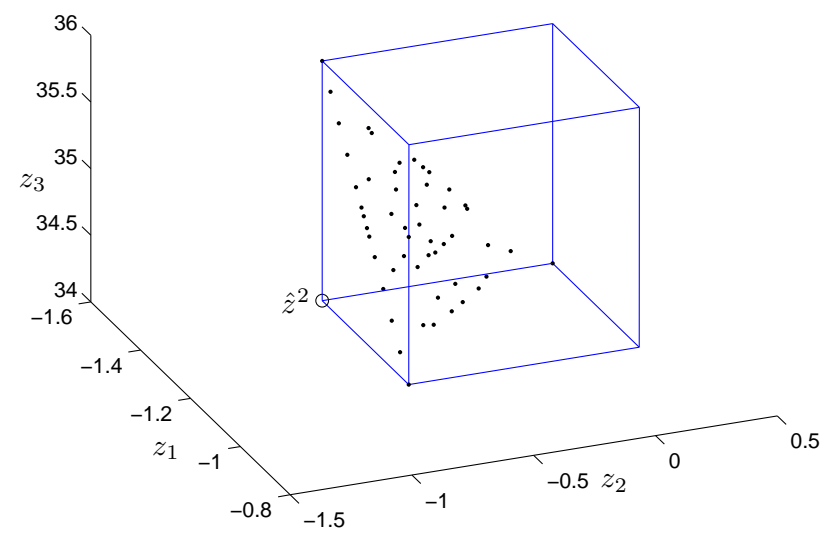

Figure 14: Third approximation of problem (8) with 50 nondominated points, considering only the first three objectives

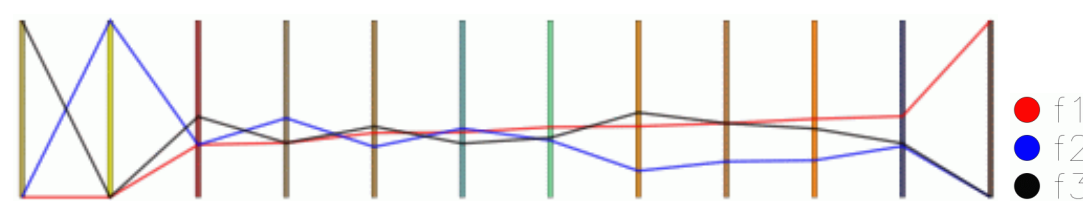

Figure 15: The first 12 nondominated points that were generated in Figure 14, illustrated by value paths

value paths in Figure 15. This information may be sufficient for the decision maker in order to select, for example, the eighth solution (from left) illustrated in Figure 15 as the most preferred solution.

Let us next consider problem (8) (with five objective functions) and set $P=$ $15(=5 \cdot 3)$. In other words, we generate 15 nondominated solutions for each approximation. To aid the decision maker in finding the most preferred solution in this five criteria case, we illustrate the solutions in each iteration using value paths. The decision maker may, in addition, select some of these solutions for further visualizations as shown, for example, in Figure 3.

Starting again with an approximation of the nadir point (obtained using a pay-off table), the 15 points that were generated in the first approximation are illustrated in Figure 16. The fourth, eleventh and the last solution (from left) are further visualized in Figure 3. Having this information available, the decision maker may, for example, select the fourth solution shown in Figure 16 to zoom into the approximation with a zooming factor of $\beta=\frac{1}{2}$ as described in Section 5.1. The recomputed approximation is again illustrated using value paths, see Figure 17. 


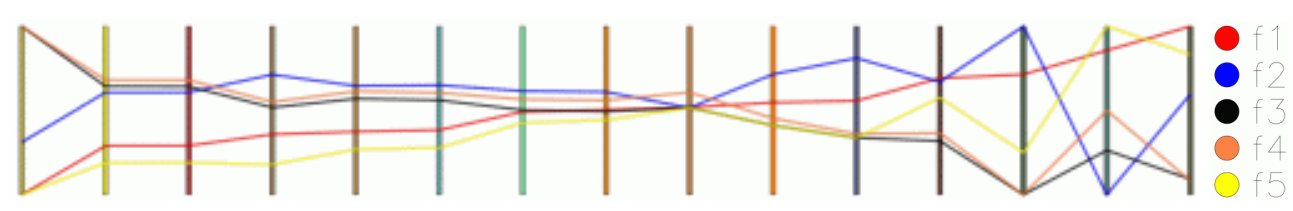

Figure 16: Value paths for the initial approximation of problem (8) with 15 nondominated points, considering all five objectives

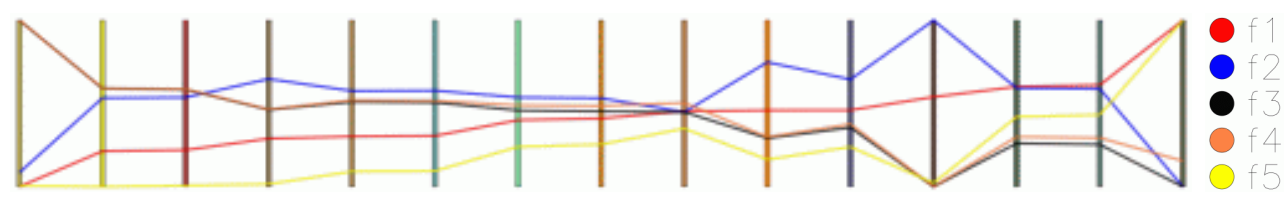

Figure 17: Value paths for the second approximation of problem (8) with 15 nondominated points, considering all five objectives

Now the decision maker may wish to zoom in more closely towards the fifth solution shown in Figure 17, using a zooming factor of $\beta=\frac{2}{3}$. The recomputed approximation is visualized in Figure 18, and the decision maker may, for example, select the tenth solution (from left) as the most preferred solution. Let us point out that the visualizations given in Figure 3 could be used for any set of solutions that the decision maker finds desirable.

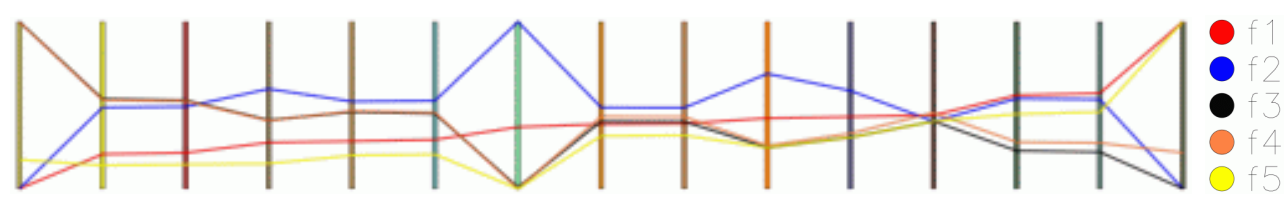

Figure 18: Value paths for the third approximation of problem (8) with 15 nondominated points, considering all five objectives

\section{Conclusions}

We have introduced a new interactive hybrid multicriteria optimization method for both convex and non-convex problems where rough approximations of the nondominated set and reference points specified by a decision maker are combined. In this approach we hybridize ideas of a posteriori and interactive methods. The advantage of this new interactive learning-oriented method is that the decision maker gets an impression of the behavior of the problem with the help of approximations and can learn about the problem without having to consider and compare too many solutions at a time. Then (s)he can identify the most interesting region of criterion values conveniently in the form of minimal acceptable criterion values (forming a reference point). The approximation is refined only 
in such specific areas that the decision maker is interested in. The calculations involved with approximations are fast and computationally efficient. After the desirable region has been identified, the decision maker can fine-tune the solution with existing interactive methods that operate with the original multicriteria optimization problem (and not the approximation) so that consideration is restricted to the interesting region found. In this way, the decision maker can find any desirable nondominated solution faster.

In the suggested new interactive algorithm, the number of optimization problems to be solved during the whole solution process can stay relatively small emphasizing the computational efficiency of the approach. This is explained both by the fact that only such parts of the nondominated set that the decision maker is interested in are studied more closely and because the current approximation can be utilized in many cases and it is then only augmented by new solutions. In other words, the approximation does not have to be calculated from scratch but the existing one can be taken as a part of the new approximation. The usability aspect is also important with interactive methods and here the information given to and asked from the decision maker is easily understandable in the form of criterion values. In other words, not too much cognitive burden is set on the decision maker.

\section{Acknowledgments}

This work was supported in part by the National Technology Agency of Finland (project Multiobjective Optimization and Multidisciplinary Decision Support). The authors wish to thank Mr. Vesa Ojalehto for his help with some of the figures utilizing the IND-NIMBUS software.

\section{References}

Ballestero, E. and Romero, C. (1998). Multiple Criteria Decision Making and its Applications to Economic Problems. Kluwer Academic Publishers, Boston.

Chankong, V. and Haimes, Y. Y. (1983). Multiobjective Decision Making: Theory and Methodology. Elsevier Science Publishing, New York.

Cohon, J. L. (1978). Multiobjective Programming and Planning. Academic Press, New York.

Edelsbrunner, H. (1987). Algorithms in Combinatorial Geometry. SpringerVerlag, Berlin.

Fandel, G. (1972). Optimale Entscheidung bei Mehrfacher Zielsetzung. Lecture Notes in Economics and Mathematical Systems 76. Springer-Verlag, Berlin. 
Geoffrion, A. M. (1968). Proper efficiency and the theory of vector maximization. Journal of Mathematical Analysis and Applications, 22(3), 618-630.

Hwang, C. L. and Masud, A. S. M. (1979). Multiple Objective Decision MakingMethods and Applications: A State-of-the-Art Survey. Springer-Verlag, Berlin.

Kamenev, G. K. (1992). A class of adaptive algorithms for the approximation of convex bodies by polyhedra. Zh. Vychisl. Mat. Mat. Fiz., 32(1), 136-152. In Russian; English translation in Comput. Maths. Math. Phys. 32(1), 114-127.

Kamenev, G. K. (1994). Investigation of an algorithm for the approximation of convex bodies. Zh. Vychisl. Mat. Mat. Fiz., 34(4), 608-616. In Russian; English translation in Comput. Maths. Math. Phys. 34(4), 521-528.

Klamroth, K. and Tind, J. (2006). Constrained optimization using multiple objective programming. Technical report, Institute of Applied Mathematics, University of Erlangen-Nuremberg. Submitted to Journal of Global Optimization.

Klamroth, K., Tind, J., and Wiecek, M. M. (2002). Unbiased approximation in multicriteria optimization. Mathematical Methods of Operations Research, 56, $413-437$.

Korhonen, P. and Laakso, J. (1986). A visual interactive method for solving the multiple criteria problem. European Journal of Operational Research, 24, $277-287$.

Lotov, A. V., Bushenkov, V. A., and Kamenev, G. K. (2004). Interactive Decision Maps. Approximation and Visualization of Pareto Frontier. Kluwer.

Miettinen, K. (1999). Nonlinear Multiobjective Optimization. Kluwer, Dordrecht.

Miettinen, K. (2003). Graphical illustration of Pareto optimal solutions. In T. Tanino, T. Tanaka, and M. Inuiguchi, editors, Multi-Objective Programming and Goal Programming: Theory and Applications, pages 197-202, Berlin, Heidelberg. Springer-Verlag.

Miettinen, K. and Mäkelä, M. M. (1999). Comparative evaluation of some interactive reference point-based methods for multi-objective optimisation. Journal of the Operational Research Society, 50, 949-959.

Miettinen, K. and Mäkelä, M. M. (2000). Interactive multiobjective optimization system WWW-NIMBUS on the Internet. Computers \& Operations Research, 27, 709-723. 
Miettinen, K. and Mäkelä, M. M. (2006). Synchronous approach in interactive multiobjective optimization. European Journal of Operational Research, 170, 909-922.

Miettinen, K., Lotov, A. V., Kamenev, G. K., and Berezkin, V. E. (2003). Integration of two multiobjective optimization methods for nonlinear problems. Optimization Methods and Software, 18, 63-80.

Nakayama, H. (1995). Aspiration level approach to interactive multi-objective programming and its applications. In P. Pardalos, Y. Siskos, and C. Zopounidis, editors, Advances in Multicriteria Analysis, pages 147-174. Kluwer, Dordrecht.

Pascoletti, A. and Serafini, P. (1984). Scalarizing vector optimization problems. Journal of Optimization Theory and Applications, 42, 499-524.

Raith, A. (2005). Bicriteria Optimization of Synchronous Generators for Wind Power Plants. Master's thesis, Technical University of Darmstadt.

Rote, G. (1992). The convergence rate of the sandwich algorithm for approximating convex functions. Computing, 48, 337-361.

Ruzika, S. and Wiecek, M. M. (2005). A survey of approximation methods in multiobjective programming. Journal of Optimization Theory and Applications, 126, 473-501.

Sawaragi, Y., Nakayama, H., and Tanino, T. (1985). Theory of Multiobjective Optimization, volume 176 of Mathematics in Science and Engineering. Academic Press, Inc., Orlando, FL.

Schandl, B., Klamroth, K., and Wiecek, M. M. (2001). Norm-based approximation in bicriteria programming. Computational Optimization and Applications, 20, 23-42.

Schandl, B., Klamroth, K., and Wiecek, M. M. (2002a). Introducing oblique norms into multiple criteria programming. Journal of Global Optimization, 23, 81-97.

Schandl, B., Klamroth, K., and Wiecek, M. M. (2002b). Norm-based approximation in multicriteria programming. Computers and Mathematics with Applications, 44, 925-942.

Statnikov, R. B. and Matusov, J. B. (1995). Multicriteria Optimization and Engineering. Chapman \& Hall, New York.

Steuer, R. E. (1986). Multiple Criteria Optimization: Theory, Computation, and Application. Wiley \& Sons, New York. 
Szidarovszky, F., Gershon, M. E., and Duckstein, L. (1986). Techniques for Multiobjective Decision Making in Systems Management. Elsevier Science Publishing, New York.

Trinkaus, H. L. and Hanne, T. (2005). knowcube: a visual and interactive support for multicriteria decision making. Computers \& Operations Research, 32, 12891309 .

Wierzbicki, A. P. (1986). On the completeness and constructiveness of parametric characterizations to vector optimization problems. OR Spectrum, 8, 73-87. 